\title{
VII. Ueber das Mineralvorkommen zu Haddam Neck in Connecticut (Ver. St.. ${ }^{1}$ ).
}

Von

H. L. Bowman in Oxford.

(Hierzu Taf. III.)

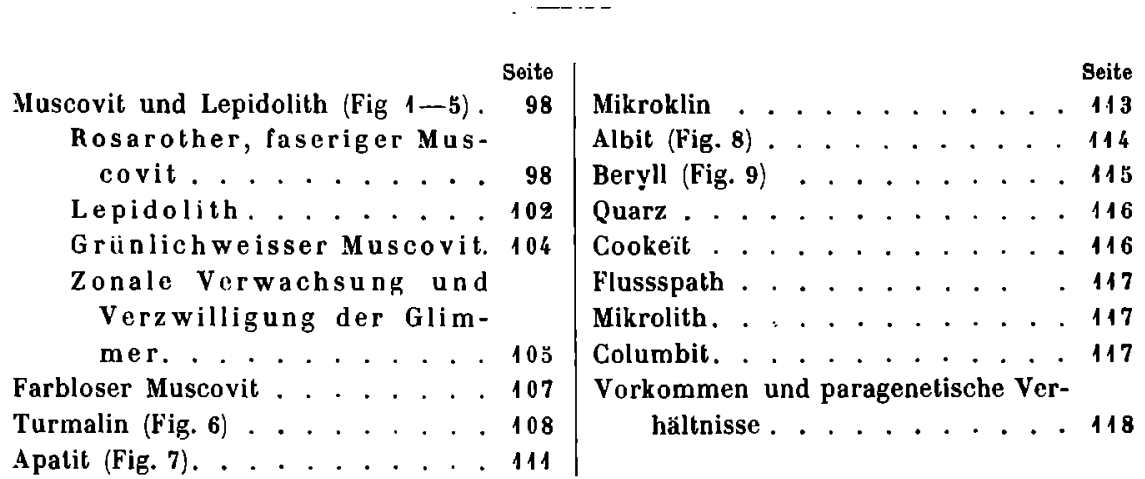

Im Laufe des Jahres 1897 gelangte (als werthvolles Geschenk des Herrn Ernest Schernikow in New York) in das mineralogische Museum zu Oxford eine reiche Sammlung von etwa 80 Stufen aus einem damals neu eröffneten Feldspathbruche in der Nähe von Haddam Neck in Connecticut.

Die in dieser Sammlung enthaltenen Mineralarten sind folgende: Grüner und rother Turmalin, Albit, Mikroklin, grüner und rosa Apatit, brauner Flussspath, Beryll, Quarz, Cookeït, lila Lepidolith, farbloser und grünlichweisser Muscovit, sowie eine ganz eigenthümliche rosafarbene faserige Varietät desselben Minerals. Diesen sind auch folgende Mineralien, da sie an demselben Orte gefunden worden sind, hinzuzufügen: grüner Flussspath, Mikrolith, Columbit.

Als Fundort wird "Haddam Neck" angegeben. Dasselbe liegt am üstlichen Ufer des hier etwa 800 Meter breiten Connecticutflusses, gegenüber Haddam. Das Vorkommen hat Herr Schernik ow mit folgenden Worten beschrieben:

1) Aus dem Min. Mag. 1902, 12, 97-121, unter Hinzufügung einiger späterer Beobachtungen und Verbesserungen, vom Verf. mitgetheill.

Groth, Zeitschrift f. Krystellogr. XXXVII. 
"The deposit is what I would call a vein of very coarse granite that is, the quartz, felspar, and mica are in very large pieces. Quartz and felspar predominate, mica being also quite abundant, and sometimes in plates 2 feet across. The tourmalines and associated minerals, like lepidolite, beryl, cookeite etc., occur usually in pockets, all of which are lined with crystallised quartz and felspar, and sometimes beryl. All the minerals occur in these pockets. The deposit is well defined between walls of country-rock - possibly gneiss, but I am not positive. *

Bei der vorliegenden Beschreibung der Arten sind die Aufstellung der Krystalle und die Buchstabenbezeichnung der Flächen stets dieselben, wie sie Dana in seinem "System of Mineralogy ( 1892) gebraucht hat. Die Dana'schen Axen sind ebenfalls angenommen worden, ausser im Falle des Turmalin, bei welchem die Flächen auf das rhomboëdrische Axensystem von Miller bezogen sind. Bei dem hexagonalen Apatit und Beryll sind auch die von Miller (in seiner Auflage der Phillips'schen "Mineralogie*, 1852) gebrauchten Buchstaben und Symbole beigefügt.

\section{Die Glimmer.}

\section{Verwachsung von Muscovit und Lepidolith.}

Die interessantesten Mineralien des ganzen Vorkommens sind wohl die Glimmer. Es werden dicke, hexagonale resp. rhombische Säulen mit der Basis gefunden, in Association mit Rauchquarz, Albit, Mikrokdin, Cookeït und zuweilen Turmalin. Dieselben besitzen gewöhnlich einen Durchmesser von 1,5 bis 3 resp. $4 \mathrm{~cm}$, aber (wenn man aus einem einzigen tafeligen (gespaltenen) Krystalle schliessen darf) erreichen gelegentlich auch $8 \mathrm{~cm}$. Sie sind an den Enden meist von Spaltungsflächen begrenzt, jedoch haben einige immer noch eine Länge von $4 \mathrm{~cm}$. Aus quergespaltenen Proben ersieht man, dass die Säulen entweder einfach aus durchsichtigen lila Lepidolith bestehen, oder (gewöhnlicher) aus einem Saume dieses Minerals und einem von demselben umhüllten Kerne von grünlichweissem Muscovit aufgebaut sind, wobei die Spaltungsrichtung ununterbrochen durch Saum und Kern läuft.

\section{Rosarother faseriger Muscovit.}

Die Oberfläche der Süulen bietet ein eigenthümliches faseriges Aussehen wegen des Vorhandenseins einer dünnen Schicht nach der Längsrichtung laufender Fasern eines rosafarbigen Minerals. Auch an den Enden wohlerhaltener Exemplare ist der Lepidolith mit einer dickeren Schicht desselben Materials, welche die Dicke von $1 \mathrm{~cm}$ überschreiten kann, bedeckt. Die Fasern liegen stets parallel der Längsrichtung der Säulen und zeigen eine vollkommene Spaltbarkeit in der Querrichtung, vermöge welcher Blättchen von beliebiger Dicke, parallel der Spaltungsrichtung des Lepidolith, 
leicht erhalten werden künnen. Derartige Blättchen bilden prächtige Objecte unter dem Mikroskope und erscheinen dann als aus kleinen rhombischen Täfelchen bestehend, mit Winkeln von $60^{\circ}$ und $120^{\circ}$, welche nach den Diagonalen auslöschen. Diese Täfelchen sind zu einer ganz regelmässigen Structur angeordnet, und zwar in dreierlei Stellung, so dass sie sich zu mosaikartigen Partien vereinigen, welche in drei von einander um je $120^{\circ}$ abweichenden Lagen Auslöschung zeigen (s. Taf. III, Fig. 1). Im convergenten Lichte zeigen die Rhomben eine ungefähr normal-austretende negative Mittellinie eines ziemlich grossen Axenwinkels, wobei die Axenebene parallel der grösseren Diagonale liegt.

Diese Eigenschaften wiesen auf ein der Glimmergruppe angehörendes Mineral, und so war eine Analyse nöthig, um die Art näher zu bestimmen.

Das für die Analyse dienende Material besass eine gleichmässige blassrosarothe Farbe, war (nach der Beobachtung unter dem Mikroskope) ganz rein und frei von fremden Einschlüssen und entbehrte jeder Spur von braunem zersetzten Material, wie es auf der Oberfläche einiger Proben vorhanden war. Die Dichte (vor dem Zerdrücken) war 2,791.

Beim Erhitzen des trockenen Pulvers in cinem Kölbchen wurde eine geringe Menge Wasser ausgetrieben, und durch qualitative Prüfung wurde noch das Vorhandensein von $\mathrm{SiO}_{2}, \mathrm{Al}_{2} \mathrm{O}_{3}, \mathrm{Fe}_{2} \mathrm{O}_{3}$ (resp. $\mathrm{FeO}$ ), $\mathrm{MnO}, \mathrm{K}_{2} \mathrm{O}, \mathrm{Na}_{2} \mathrm{O}, \mathrm{Li} \mathrm{O}_{2} \mathrm{O}, \mathrm{F}, \mathrm{P}_{2} \mathrm{O}_{5}$ nachgewiesen. Das Manganoxydul genügt, um den geschmolzenen Alkalicarbonaten eine blasse, bläulichgrüne Farbe zu geben, und ist vielleicht die Ursache der Rosafarbe des Minerals, es scheint aber in wägbarer Menge nicht vorhanden zu sein.

Das feingericbene Mineral zeigte nach 48 stündigem Stehen über Chlorcalcium fast keinen, und nach zweistündiger Erhitzung bei $96^{\circ} \mathrm{C}$. in einem Trockenkasten einen nur ganz geringen Gewichtsverlust. Für die quantitativen Bestimmungen wurde daher das Material ouf letztere Weise getrocknet.

Das Wasser wurde durch die Penfield'sche directe Methode bestimmt 1), wobei das mit vorher gut getrocknetem, zum Zurückhalten des Fluors dienenden Bleioxyd gemischte Mineral in einem am Ende zugeschmolzenen Rohre aus Kaliglas mit einem Bunsen' schen Gasbrenner erhitzt wurde. Das Wasser wurde in einer am oberen Theile des Rohres angeblasenen, mit nassem Löschpapier bedeckten Kugel condensirt. Die das Wasser enthaltende Kugel wurde abgeschnitten und gewogen, und dann nach dem Austrocknen des (neutral reagirenden) Wassers nochmals gewogen. Eine weitere Erhitzung des Mineralrückstandes in der Gebläseflamme lieferte kein Wasser mehr. In zwei Versuchen ergaben $0,0975 \mathrm{~g}$ resp. $0,0759 \mathrm{~g}$ des trockenen Minerals $0,0043 \mathrm{~g}$ resp. $0,0033 \mathrm{~g}$ Wasser, entsprechend $4,41 \%$ resp. 4,35\% Wasser [im Mittel 4,38\%].

Die Bestimmung des Fluors geschah durch die bekannte Berzelius'sche Methode. Zwei Versuche mit $0,1186 \mathrm{~g}$ resp. $0,2531 \mathrm{~g}$ des trockenen Minerals ergaben (nach Abzug von $\frac{1}{T_{0}}$ der Lösung für die Prüfung auf Phosphorsäure) $0,0008 \mathrm{~g}$ resp. $0,0018 \mathrm{~g} \mathrm{CaF}_{2}$, welche einer tolalen procentischen Menge von 0,37 resp. 0,385 Fluor entsprechen [Mittel $0,377 \%$ ].

Wegen unbefriedigender Resultate bei der Kieselsäurebestimmung mit dem Berzelius'schen Verfahren wurde dieser Bestandtheil auf gewöhnlichem Wege bestimmt

1) Amer. Journ. Sc. 1894, (3), 48, 30. 
durch Abdampfen der Carbonatschmelze mit Säure Etwaiger von Fluor verursachter Verlust an Kieselsäure wird, bei so wenig vorhandenem Fluor, nur unbedeutend sein.

Sieben Versuche ergaben die unten folgenden Zahlen.

Die saure Lösung wurde in jedem Falle zweimal im Wasserbade abgedampft und durch zwei verschiedene Filter abfiltrirt. Ferner wurde in Nr. 3, 4, 5, 6 der Rückstand nach der zweiten Abdampfung bei $110^{0}$ in einem Trockenkasten ${ }^{1)}$ erhitzt, bis keine sauren. Dämpfe mehr entwichen. In $\mathrm{Nr}$ 3, 4, 7 wurde $\mathrm{HNO}_{3}$ statt $\mathrm{HCl}$ benutzt.

Die gewogene Kieselsäure wurde im offenen Platintiegel $12-13$ Std. den Dämpfen

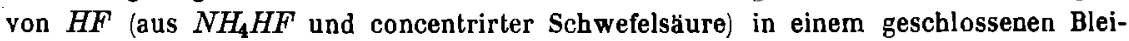
kasten 1) über einer kleinen, ziemlich tief unten gelegenen Flamme ausgesetzt. Die Kieselsäuremenge wurde dann aus dem Gewichtsverluste erhalten.

$\begin{array}{lcccccccc} & 1 . & 2 . & \mathbf{3 .} & 4 . & \mathbf{5 .} & \mathbf{6 .} & 7 . & \text { Mittel : } \\ \text { Gew. Mineral } & \mathbf{0 , 3 8 2 7} & \mathbf{0 , 2 4 2 3} & \mathbf{0 , 3 0 2 3} & \mathbf{0 , 3 2 0 8} & \mathbf{0 , 3 0 6 9} & 0,3045 & \mathbf{0 , 3 0 0 4} & - \\ \text { Gew. } \mathrm{SiO}_{2} & 0,1792 & \mathbf{0 , 1 1 1 2} & \mathbf{0 , 1 3 9 9} & \mathbf{0 , 1 4 7 5} & \mathbf{0 , 1 4 1 9} & \mathbf{0 , 1 4 0 9} & \mathbf{0 , 1 3 9 6} & - \\ \text { Procent } \mathrm{SiO}_{2} & 46,83 & 45,89 & 46,28 & 45,98 & 46,24 & 46,27 & \mathbf{4 6 , 4 7} & 46,28\end{array}$

Das Filtrat von der Kieselsäure in Nr. 1, 2, 6 wurde in der Hitze mit Ammoniak gefällt, dann wurde der Niederschlag gewaschen, mit $B N O_{\mathfrak{a}}$ aufgelöst und wieder heiss gefällt. Der gewogene Nicderschlag von $\mathrm{Al}_{2} \mathrm{O}_{3}, \mathrm{Fe}_{2} \mathrm{O}_{3}$ und $\mathrm{P}_{2} \mathrm{O}_{5}$ wurde durch Schmelzen mit Alkalicarbonaten auf Manganoxydul geprüft, ergab aber kaum eine grüne Färbung. Die Niederschläge wogen $0,1456,0,0917,0,1153 \mathrm{~g}$, entsprechend 38,05 , $37,85,37,87 \%\left(\mathrm{Al}_{2} \mathrm{O}_{3}+\mathrm{Fe}_{2} \mathrm{O}_{3}+\mathrm{P}_{2} \mathrm{O}_{5}\right)\left[\right.$ im Mittel $\left.37,92 \% \%_{0}\right]$.

Das Filtrat von $\mathrm{SiO}_{2}$ in $\mathrm{Nr} .5$ und in einem zweiten Versuche mit $0,3070 \mathrm{~g}$ trockenem Minerale wurde mit Ammoniak gefällt und der Nicderschlag gewaschen, mit schwacher Schwefelsăure aufgelöst, dann das Eisen mit Zink reducirt und mit Kalipermanganat litrirt. Dic Resultate ergaben 1,07 resp. 0,87\% $\mathrm{Fe}_{2} \mathrm{O}_{3}$. Das Mineral wurde nicht auf Eisenoxydul besonders untersucht [Mittel $0,97 \% \mathrm{Fe}_{2} \mathrm{O}_{3}$ ].

Aus dem Filtrat in Nr. 7 wurde die Phosphorsäurc mit Ammoniakmolybdat gefällt und dann als $\mathrm{Mg}_{2} \mathrm{P}_{2} \mathrm{O}_{7}$ gewogen. Daraus ergab sich $0,09 \% \mathrm{P}_{2} \mathrm{O}_{5}$.

Aus den Mitteln der Bestimmungen ergiebt sich durch Subtraction $\mathbf{3 6 , 8 6 \%} \% \mathrm{Al}_{2} \mathrm{O}_{3}$.

Für die Alkalibestimmung wurde das Mineral nach dem Verfahren von $L$ aw rence Smith zersetzt, worauf die Trennung des Lithium nach der von Gooch2) vorgeschlagenen Methode mit Amylalkohol ausgeführt wurde. Das Lithium wurde als $\mathrm{Li}_{2} \mathrm{SO}_{4}$ gewogen. Im Rückstande wurde das Kalium von Natrium als $\mathrm{K}_{2} \mathrm{PtCl} 6$ getrennt. Der Niederscblag wurde dann geglüht und das so erhaltene Platin gewaschen und gewogen. Das Natrium wurde aus der Differenz bestimmt.

Drei Versuche mit 0,3096, 0,3049 resp. 0,3986 g trockenem Mineral ergaben (nach passender Correction für die Löslichkeit) folgende procentische Zahlen:

\begin{tabular}{lrrrr} 
& 1. & \multicolumn{1}{c}{ 2. } & \multicolumn{1}{c}{. } & Mittel: \\
$\mathrm{Li}_{2} \mathrm{O}$ & 0,31 & $\mathbf{0 , 2 5}$ & 0,21 & 0,26 \\
$\mathrm{~K}_{2} \mathrm{O}$ & 10,90 & 10,53 & 10,46 & 10,63 \\
$\mathrm{Na}_{2} \mathrm{O}$ & 1,37 & 1,52 & 1,33 & 1,41
\end{tabular}

1) Ein derartiger Kasten kann auf folgende Weise bequem construirt werden. Man nimmt ein Stück Bleirohr von etwa $10 \mathrm{~cm}$ Durchmesser und desgleichen Länge und feilt darauf die Enden plan und glatt. Der Boden und der Deckel des Kastens bestehen aus zwei losen planen Platten aus starkem Bleiblech.

Der Tiegel wird mit einem kleinen Dach aus Platinblech bedeckt, um die Kieselsäure von etwaigen vom Deckel des Kastens herabfallenden Partikeln zu schützen.

2) Proc. Amer. Acad. Arts and Sc. 1886, N. S., 14, 177; Bull. U. S. Geol. Survey 1887, Nr. 42, 73; Chem. News 1887, 55, 18 etc. 
Die Zusammensetzung des Minerals kann daher auf folgende Weise ausgedrückt werden :

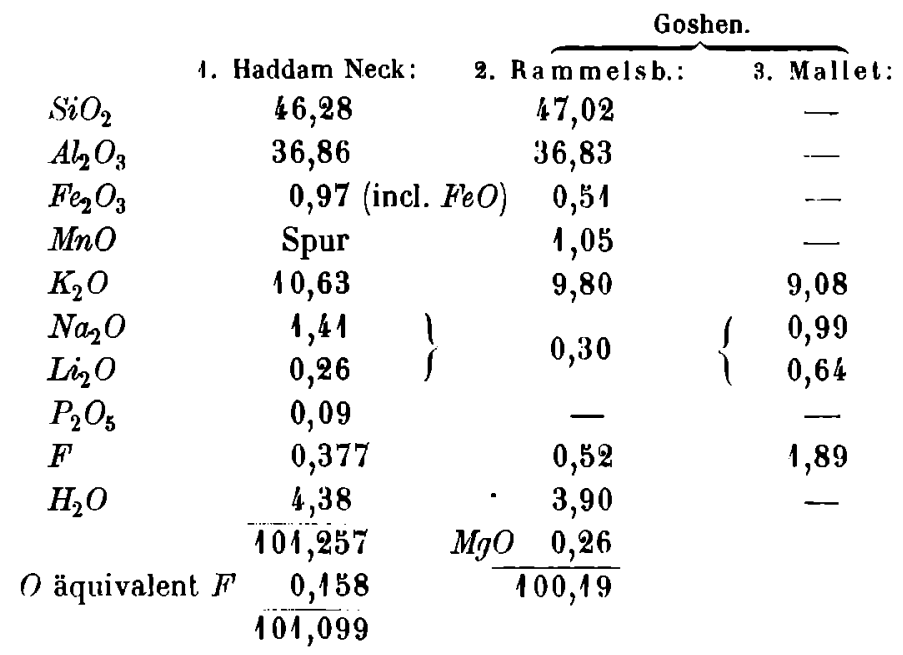

Das Mineral gehört daher zum Muscovit und ist mit dem rosarothen Muscovit von Goshen (Massachusetts) zu vergleichen. Eine Analyse des letzteren von Rammelsberg ${ }^{1}$ )., sowie eine Alkalibestimmung desselben von Mallet ${ }^{2}$ ) 3. sind oben beigefügt.

Die Gestalt einer kleinen, von der Seite einer Lepidolithsäule gesonderten Faser wurde durch goniometrische Messung als eine sehr spitze Pyramide bestimmt (Fig. 2, Taf. III). Die anscheinend verticalen Flächen liegen also nicht in einer Zone ${ }^{3}$ ). Die Winkel sind:

\begin{tabular}{|c|c|}
\hline Gemessen : & Berechnot: \\
\hline$S S=59^{0} 52^{\prime}$ & $59^{\circ} 58^{\prime}$ \\
\hline$\pi \pi=5943$ & 5952 \\
\hline$c S=8821 \frac{1}{2}, 88^{\circ} 21^{\prime}$ & $8836 \frac{1}{2}$ \\
\hline$c \pi=8638, \quad 8633$ & 8628 \\
\hline
\end{tabular}

Die Symmetrieebene halbirt demnach die stumpfen Winkel des Rhombus. Die gemessenen Winkel deuten auf die Indices $S\{\overline{5} 51\}$ und $\pi\{552\}$. Die beiden Formen sind für den Muscovit neu; $S\{\overline{5} 51\}$ wird aber von Dana für den Biotit angegeben. Den entsprechenden, oben angeführten, berechneten Werthen sind die von Dana für den Muscovit angenommenen Tschermak'schen Axen $\left(a: b: c=0,57735: 1: 3,3128 ; \beta=89^{0} 54^{\prime}\right)$ zu Grunde gelegt.

1) Min. Chem. $1875,514$.

2) Amer. Journ. Sci. 1857 (2), 23, 180.

3) Die Abweichung von der Gestalt eines Prismas ist (der Deutlichkeit halber) in Fig. 2 etwas übertrieben. 
Der optische Axenwinkel in Luft ist:

$$
2 E=75 \frac{1}{2}^{\circ}(\mathrm{Na} \text {-Licht). }
$$

Dispersion $\varrho>v$ (gering).

Die spitze Bisectrix erscheint manchmal nicht genau in der Mitte des Gesichtsfeldes; doch entspricht dies (wie es mir erst in den letzten Tagen nachzuweisen gelungen ist) ${ }^{1}$ ) nicht einer wirklichen Neigung der Bisectrix zur Plattennormale, sondern rührt einfach von der unebenen Beschaffenheit der aus mehreren Rhomben zusammengesetzten Präparate her.

Einige der Spaltungsblättchen zeigen eine helle, nirgends auslöschende Linie zwischen den in gegenseitiger Zwillingsstellung befindlichen Partien, welche von der zur Spaltungsebene nicht senkrechten Lage der Anwachsflächen herrührt.

Wegen seiner mosaikartigen Structur scheint das Mineral dem blossen Auge undurchsichtig, obwohl die einzelnen Fasern für sich vollkommen durchsichtig sind. Der Durchmesser der rhombischen Einzelkrystalle ist in der Regel ziemlich gleichmässig, und zwar etwa $0,18 \mathrm{~mm}$ nach der grösseren Diagonale. Einige wenige derselben erreichen jedoch $0,65 \mathrm{~mm}$.

Lange Zeit konnte ich keine mit diesem faserigen Muscovit überhaupt vergleichbaren, von anderen Fundorten stammenden Exemplare finden. Doch schliesslich entdeckte ich unter den Lepidolithen im Wiener Hofmuseum einen einzigen grossen tafeligen Krystall von Muscovit, welcher von einem Saume von lila Lepidolith umgeben war und am äusseren Rande des letzteren eine kleine Menge rhombisches, mosaikartiges Material zeigte. Der Krystall stammt von Auburn, Maine, und zeigte sich bei näherer Untersuchung als denjenigen von Haddam Neck sehr ähnlich. Die Rhomben befinden sich in Zwillingsstellung und löschen $120^{\circ}$ von einander aus. Die Axenebene ist der grösseren Diagonale parallel, mit ziemlich grossem Axenwinkel $\left(2 E=75^{\circ} 20^{\prime}\right)$ und deutlicher Dispersion $(\varrho>v)$. Im Lepidolithsaume liegt die Axenebene parallel einem Strahle der Schlagfigur; dieser Glimmer ist also brachydiagonal. Er ist vielfach verzwillingt. Der Muscovitkern hat die Axenebene makrodiagonal, den Axenwinkel ziemlich gross mit beträchtlicher Dispersion in dem Sinne $\varrho>v$. Der ganze Krystall ist dem oben erwähnten, grossen tafelförmigen Krystalle von Haddam Neck sehr ähnlich.

\section{Lepidolith.}

Die sechsseitigen Säulen von Lepidolith, sowie die Muscovitkerne umgebenden Säume stellen äusserst schöne Beispiele dieses Minerales dar. Das Material hat eine prachtvolle lila Farbe und ist vollkommen durchsichtig: Dem blossen Auge scheint es ganz gleichmässig und homogen, lässt aber

1) Durch Losmachen eines einzigen (grösseren) Rhombus und unter Anwendung des später (S. 103) zu beschreibenden Beobachtungsverfahrens. 
erst durch Inwendung der Körnerprobe und Untersuchung unter dem Mikroskope erkennen, dass die Krystalle in der That in zwei Gruppen zerfallen, bei deren einer der Glimmer brachydiagonal, bei der anderen dagegen makrodiagonal ist. Es darf hier der Thatsache Erwähnung geschehen, dass beim Lepidolith eine Schlagfigur viel schwieriger zu erzeugen ist, als beim Muscovit, weil jener mehr biegsam und weniger elastisch ist als dieser.

Der Lepidolith wird allgemein zur Gruppe der makrodiagonalen Glimmer gerechnet, doch ist eine brachydiagonale Varietät von Schüttenhofen in Böhmen von Scharizer ${ }^{1}$ ) beschrieben worden, wo die zonale Verwachsung der Glimmer sowie die Turmaline scheinbar im Allgemeinen denjenigen des vorliegenden Vorkommens ähnlich sind.

Spaltungsblättchen des Lepidolith von Haddam Neck zeigen (wenn nicht allzu dünn) in der Regel keine Auslöschung wegen des Aufeinanderliegens von Schichten in Zwillingsstellung. Sehr dünne Blättchen sind aus unregelmässigen Partien, welche $120^{\circ}$ von einander auslöschen, zusammengesetzt. Wegen der Zwillingshildung ist der $\Lambda$ xenwinkel ein schwankender; er ist aber wohl stets kleiner als beim Muscovit.

Die brachy-, sowie die makrodiagonale Varietät zeigen eine geringe Dispersion der Axen in dem Sinne $\varrho>v$. I) brachydiagonalen Blättchen zeigen eine $\Lambda$ bweichung der Mittellinie von der Plattennormale in der Ebene der optischen $\Lambda$ xen. Bei den makrodiagonalen Blättchen ist auch eine $\mathbf{A b}$ weichung der Mittellinie um etwa $54^{0}-7 \mathbf{1}_{2}{ }^{\circ}$ von der Mitte des Gesichtsfeldes. zu beobachten, und zwar wieder in der optischen Axenebene, also in diesem Falle quer zur Symmetrieebene der Glimmer. Da nun eine derartige Abweichung der Annahme monokliner Symmetrie widersprechen würde, war es von höchster Wichtigkeit festzustellen, ob die Erscheinung auf einer Thatsache oder in irgend welchem Fehler des Apparates (wie z. B. in einer ungenauen Justirung der Axe des Mikroskopes senkrecht zum Objecttische), resp. in einer schiefen Lage des allerdings etwas unebenen Blättchens auf dem $0 \mathrm{~b}-$ jectträger begründet war.

Es wurde zu diesem Zwecke ein kleines und besonders ebenes Blättchen ausgewählt und dann so auf einem dünnen Deckgläschen aufgekittet, dass bei der Beobachtung auf dem Goniometer der beste vom Glimmer gegebene Reflex von dem vom Gläschen gelieferten nur um einige wenige Minuten abwich. Die Partien des Glimmers, welche andere Bilder gaben, wurden mit einer Papiermaske bedeckt. Das so präparirte Blättchen wurde dann auf eine Scheibe aus planparallelem Glase aufgelegt, und letztere auf den Objecttisch eines (mit drehbaren Nicols versehenen) Mikroskopes so aufgesetzt, dass sie innerhalb eines Pappringes in ihrer eigenen Ebene gedreht werden konnte. Die Lagen der beiden optischen Axen $\left(O_{1}, O_{2}\right)$ im

1) Diese Zeitschr. 1887, 18, 1. 
Gesichtsfelde wurden auf einem Ocularmikrometer abgelesen, zunächst in der einen Stellung $(\Lambda)$ und dann nach einer Drehung der Scheibe auf dem Objecttische um $180^{\circ}$ (Stellung B). Das Mittel von $O_{1_{\Lambda}}$ und $O_{l_{\mathrm{B}}}$ (resp. von $O_{2_{\mathrm{A}}}$ und $O_{2 \mathrm{~B}}$ ) giebt nun die Lage der Plattennormale, während durch diejenigen von $O_{1_{\Lambda}}$ und $O_{2_{\Lambda}}, O_{\mathbf{1}_{\mathrm{B}}}$ und $O_{2_{\mathrm{B}}}$ die Lagen der Mittellinien in den beiden Stellungen bestimmt werden.

Ein Blättchen, welches sich später durch die Körnerprobe als makrodiagonal erwies, zeigte eine Abweichung der Mittellinie von der Plattennormale von $61_{2}^{\circ}$ in der Ebene der Axen, während die Neigung des Glimmers zum Deckgläschen nur $16^{\prime}$ betrug. Die wirkliche Neigung der Mittellinie zur Normale zur Spaltungsebene in der Ebene der optischen Axen kann demnach nicht weniger als $64^{0}$ sein.

Daraus muss man den Schluss ziehen, dass, wenn ein Strahl der Schlagfigur ${ }^{1)}$ nicht senkrecht zur Ebene (010) liegt, dieser Glimmer dem triklinen Systeme angehört.

Bei der Auslöschungsstellung der Nicols fällt eine etwaige seitliche, durch Drehung der Scheibe um $1 \mathbf{8 0}^{\circ}$ hervorgebrachte, Verschiebung des durch die beiden Axen gehenden Balkens innerhalb der Beobachtungsfehler. Die Axenebene ist also merklich senkrecht zur Spaltungsebene.

Ein zweites Blättchen, welches sich als brachydiagonal erwies, auf ohige Weise untersucht, zeigte eine Neigung der Mittellinie von $4 \frac{30}{4}$ in der optischen Axenebene, wobei der Glimmer nirgends um mehr als $7^{\prime}$ zum Deckgläschen geneigt war.

\section{Grünlichweisser Muscovit.}

Der Kern mehrerer der Glimmersäulen besteht aus grünlich-, resp. gelblichweissem Muscovit, und ein ähnliches Material kommt auch in unregelmässig begrenzten Blättchen vor, welche durch derben Quarz in paralleler Stellung zerstreut sind.

Der Kern des oben erwähnten (S. 98) grossen tafeligen Krystalles hat einen scheinbaren Axenwinkel in Luft ${ }^{2}$ :

$$
2 E=75^{\circ} 38^{\prime} \text {. }
$$

Ein ähnliches Material aus einem kleineren Prisma mit rhombischem Umrisse hat:

$$
2 E=74^{\circ} 35^{\prime} \text {. }
$$

Dieser Glimmer ist ein vollkommen normaler Muscovit; er hat die

1) Es ist wohl nicht daran zu zweifeln, dass der Stern eine Schlagfigur (und nicht etwa eine Druckfigur) darstellt, weil seine Form gut mit dem von M. Bauer abgebildeten Typus übereinstimmt (Zeitschr. d. d. geol. Ges. 1874, 26, Taf. II, Fig. 2; auch in Hintze, Mineralogie 2, 518, Fig. 236).

2) Für den Muscovitkern des zonalen Glimmers von Schüttenhofen giebt Scharizer an: $2 E=7^{0} 52^{\prime}$ für $\mathrm{Na}$-Licht. 
Axenebene makrodiagonal mit beträchtlicher Dispersion der Axen im Sinne $\varrho>v$. Die Blätter sind ganz einheitlich und zeigen keine Zwillingsstructur.

\section{Zonale Verwacheung und Verzwilligung der Glimmer.}

1. Lepidolith auf Muscovit. Die Grenze zwischen dem Muscovitkerne und dem Lepidolithsaume ist zuweilen unregelmässig, meistentheils aber gerade und scharf. Der Umriss derselben ist gewöhnlich von entweder rhombischer oder hexagonaler Form, dem Klinopinakoid und Flächen der Zone $[001: 110]$ des Muscovits entsprechend. Die stumpfen Winkel des Rhombus sind oft von kleinen Flächen $a\{100\}$ abgestumpft und die Ecken zwischen $\{010\}$ und $\{110\}$ häufig durch kurze Kanten ersetzt, welche wahrscheinlich verschiedene Formen darstellen. Wegen der Kürze der Kanten und der unvollständigen Ausbildung der Ecken sind letztere kaum zu bestimmen, doch ist wohl $Q\{130\}$ vorhanden, und zwei Kanten, welche Winkel von $23^{0}$ resp. $24^{0}$ mit $\{110\}$ bilden, entsprechen vielleicht einer Form $\{370\}$, wofür der berechnete Winkel (wenn man $(110):(1 T 0)=60^{\circ}$ setzt) $=23^{\circ} 25^{\prime}$. Aus sieben Messungen (zwischen den Grenzen $59^{\circ}$ und 601. ${ }^{\circ}$ ) unter dem Mikroskope ergah sich 5910 als Mittelwerth des ebenen Winkels des Rhombus.

Die Beziehungen des Lepidoliths zum Muscovitkerne sind wegen der innig verzwilligten Structur des ersteren nicht leicht zu erforschen, weil die einheitlichen Partien so klein sind, dass man auf ihnen eine unverkennbare Schlagfigur nur ausnahmsweise herstellen kann.

Dicht ausserhalb der Grenze befindet sich in der Regel (aber nicht. immer, s. Fig. 4, Taf. III) ein schmales paralleles Band von Lepidolith $\left(L_{m}\right)$, welches in derselben Stellung wie der Muscovit auslöscht und die optische Axenebene parallel derjenigen des Muscovits hat (s. Fig. 3, Taf. III). Nur zweimal ist es mir gelungen, auf diesem Bande eine Schlagfigur herzustellen. Dadurch wurde aber das Material als makrodiagonaler Glimmer in paralleler Stellung zu dem Muscovit erwiesen.

Ausserhalb dieses inneren Lepidolithbandes und gelegentlich auch dicht am Muscovit sind unregelmässig begrenzte Partien $\left(L_{b}\right)$ mit der Axenebene parallel einer oder der anderen Kante des Rhombus, d. h. um $30^{\circ}$ in einer oder der anderen Richtung gegen diejenige des Muscovits gedreht (Fig. 3 u. 4).

Diejenigen Partien, auf welchen ich eine Schlagfigur erhalten konnte, gehören der brachydiagonalen Varietät an, also ist es wahrscheinlich, dass alle als brachydiagonaler Glimmer in Zwillingsstellung nach dem gewöhnlichen »Glimmergesetze * (Zwillingsebene $\{110\})$ zu erklären sind.

Die Auslöschung des Lepidoliths sowie des Muscovits in der Nähe der Grenze ist etwas schwankend und unvollkommen; daher können die Schwingungsrichtungen nicht so genau bestimmt werden, dass man daraus einen Schluss ziehen könnte, ob sich der an den Muscovit anstossende Lepidolith zu diesem oder zu dem dazu parallelen Lepidolith in Zwillingsstellung 
betindet (in den Fig. 4 entsprechenden Fällen) und ob letzteres Lepidolithband dem Muscovit genau parallel oder auf demselben unter Berührung der Prismenflächen aufgewachsen ist. Die schwankende Auslöschung ist wohl selbst ein Anzeichen der Spannung, welche durch die unvollkommene Uebereinstimmung der Molekulargerüste der beiden Mineralien hervorgebracht wird.

Bei den grösseren Krystallen besteht der Lepidolithsaum aus innig verzwilligtem Material, so dass Auslöschung fast nirgends zu beobachten ist. Jedoch in sehr dünnen Blättchen können auf vereinzelten kleinen Partien die Schwingungsrichtungen ermittelt werden. Die an die Grenze anstossenden Partien haben ihre Axenebene parallel derjenigen des Muscovits und sind wohl makrodiagonal. Andere dagegen in der Mitte des Lepidoliths, bei welchen die Axenebene einen Winkel von $30^{\circ}$ mit der Axenebene des Muscovits bildet, sind durch die Körnerprobe als brachydiagonaler Glimmer in Zwillingsstellung erwiesen.

Der äussere Umriss des Lepidoliths ist stets dessen innerer Grenze parallel, obwohl in den grösseren Krystallen er oft etwas rauh und uneben ist. Dieser Umriss muss daher verschiedene Formen darstellen je nach der Fläche, mit welcher der Lepidolith mit dem Muscovit in Berührung kommt. Also z. B. um die $\{110\}$ entsprechende Kante eines Muscovitrhombus ist der Umriss des in paralleler Stellung resp. in Zwillingsstellung nach der betreffenden Kante befindlichen Lepidoliths $\{110\}$, während bei einer Lepidolithpartie in Zwillingsstellung nach der anderen Prismenfläche Umriss sowie Berührungsfläche beide parallel $\{010\}$ sind (s. Fig. 4, Taf. III).

2. Rosarother faseriger Muscovit auf Lepidolith. Die Berührungslinie des faserigen Muscovits mit dem Lepidolith ist meist vollkommen scharf und gerade. Die kleinen Rhomben des Muscovits befinden sich entweder in paralleler Stellung auf dem Lepidolith oder sind darauf nach dem gewöhnlichen Gesetze verzwilligt. Die allgemeine Structur ist aus Fig. 5, Taf. III ersichtlich, welche ein Blättchen eines sechsseitigen Lepidolithprismas ohne Muscovitkern darstellt. Dasselbe besteht aus drei unregelmässig verzwilligten, makrodiagonalen Lepidolithkrystallen, und die Seiten des Sechseckes werden hauptsächlich aus Flächen von $\{010\}$ gebildet. An einem Individuum ist auch noch $\{110\}$ vorbanden und die Ecken sind durch kurze, $\{130\}$ entsprechende Kanten abgestumpft.

Um den brachydiagonalen Lepidolith ist die Anordnung der Rhomben ganz ähnlich, so dass die Fasern bald in paralleler, bald in Zwillingsstellung aufgewachsen sind, also mit den Axenebenen um $90^{\circ}$ resp. $30^{\circ}$ gegen diejenige des Lepidoliths gedreht.

Das Wichtigste über das oben erwähnte Glimmervorkommniss kann etwa folgendermassen zusammengefasst werden. 
Eine neue Varietät des Muscovit, welche sonst kaum vorzukommen scheint, ist hier sehr reichlich. Sie besteht aus Massen von Fasern mit rhombischem Querschnitte, welche auf die Weise in paralleler resp. Zwillingsstellung zusammengehäuft sind, dass das Ganze wie ein einheitlicher Krystall gespalten werden kann. Die Fasern sind meist um den Rand resp. an den Enden von Lepidolithsäulen gruppirt und erscheinen auf den ersten Blick einem etwaigen Umwandlungsproducte der letzteren ähnlich.

Der lilafarbige Lepidolith kommt als ganze prismatische Krystalle oder in Form eines Saumes um grünlichweissen Muscovit vor. Es giebt zwei Varietäten, welche sich nur in Bezug auf die Lage der optischen Axenebene unterscheiden, welche bei der einen Varietät zur brachydiagonalen, bei der anderen zur makrodiagonalen Axe parallel liegt. Im letzteren Falle, wegen einer Neigung der spitzen Bisectrix zur Ebene (010), muss der Glimmer dem triklinen Systeme angereiht werden. Der Muscovitkern wird allgemein von einem schmalen Saume des makrodiagonalen Lepidolith in paralleler Stellung umgeben (Fig. 3, Taf. III), während weiter nach aussen sich Partien des brachydiagonalen Lepidolith befinden, welche zu einander und zum makrodiagonalen Saume in Zwillingsstellung liegen. Darunter sind auch Partien der makrodiagonalen Yarietät zu beobachten. Brachydiagonaler I.epidolith in paralleler Stellung auf dem Muscovit, sowie makrodiagonaler Lepidolith in Zwillingsstellung auf demselben wurden nicht beobachtet. Jedoch ist brachydiagonaler Lepidolith zuweilen in directem Contact mit dem Muscovit in Zwillingsstellung zu finden (Fig. 4).

Der Lepidolith ist oft von einer dünnen Schicht rhombischer Fasern des rosarothen Muscovits in paralleler resp. Zwillingsstellung umgeben. Die Anordnung der letzteren ist um brachydiagonalen, sowie um makrodiagonalen Lepidolith die gleiche.

Die Verwachsung der beiden Lepidolitharten ist wohl bisher nicht beobachtet worden.

Sämmtliche beobachteten Zwillingsverhältnisse können auf das gewöhnliche »Glimmergesetz (Zwillingsebene (110)) zurückgeführt werden.

\section{Farbloser Muscovit.}

Ausser dem oben erwähnten grünlichweissen Muscovit, welcher Verwachsungen mit dem Lepidolith bildet, kommen auch noch grosse Blätter (bis zu $60 \mathrm{~cm}$ Durchmesser, nach Herrn Schernikow) gewöhnlichen farblosen Muscovits vor, wie er für technische Zwecke gebraucht wird. Dieselben zeigen in dickeren Blättern eine bräunliche Farbe. Sie enthalten häutige Einschlüsse eines dunklen Materials, welche im auffallenden Lichte eine bronzeartige Farbe zeigen, im durchgehenden Lichte dagegen unter dem Mikroskope undurchsichtig oder höchstens (in äusserst dünnen Häutchen) grau erscheinen. Die Einschlüsse haben oft einen hexagonalen Umriss, 
wobei die Seiten senkrecht zu den Strahlen der Schlagfigur liegen, und sind von schmalen, zu den Seiten des Sęchsecks senkrecht stehenden Rissen durchsetzt. Der Durchmesser beträgt gelegentlich $2-3 \mathrm{~mm}$, ist aber meist kleiner. Wegen der ungeheuren Dünne der Häutchen habe ich sie nicht isoliren resp. bestimmen können. Auf eine Magnetnadel haben sie scheinbar keine Wirkung.

Dieser Glimmer enthält auch flache tafelige und längliche Krystalle von schwarzem, braunem und grünem Turmalin.

Die Axenebene des Glimmers ist makrodiagonal und der Axenwinkel beträgt $71^{0} 0^{\prime}=2 E$.

\section{Turmalin.}

Der Turmalin bildet prachtvolle, durchsichtige, gestreifte Prismen von gekrümmtem dreiseitigem resp. flachem Querschnitt und von mehreren verschiedenen Farben. Unter den häufigsten Farben sind Nüancen von hellem und dunklem Grün und Blassroth ${ }^{1)}$ zu nennen. Einige wenige Krystalle sind fast vollkommen farblos. Die Säulen zeigen oftmals buntfarbige Querbänder, deren zum Prisma senkkrecht stehende Grenzebenen bald vollkommen glatt und scharf, bald etwas wolkig erscheinen. Einmal hat die Grenze zwischen einem blassgrünen und einem röthlichen Bande die Form der trigonalen Pyramide $\{11 T\}$ (Fig. 6, Taf. III) und zuweilen wird das pyramidale Ende $\{11 T\}$ aus einer die gleichgestaltete frühere Termination bedeckenden, parallelen Schicht von einer verschiedenen Farbe gebildet. Merkwürdig ist die Häufigkeit des Alleinvorkommens der Basis unter diesen Grenzebenen, weil gerade diese Form bei den jetzigen Terminationen der Krystalle meist zurücktritt. Zuweilen ist eine Säule theilweise von einem Bande aus verschieden gefärbtem Material umhüllt. Die vorliegenden Krystalle zeigen also keine Aehnlichkeit mit den bekannten (schwarzen) Turmalinen von $\times$ Haddam *.

Die Krystalle sind manchmal doppelendig; ihr Habitus schwankt von kurzen, dicken Säulen $(30 \mathrm{~mm}$ lang $\times 20 \mathrm{~mm}$ Durchm.) bis auf lange, schlanke Prismen $\left.(80 \mathrm{~mm} \times 4 \mathrm{~mm})^{2}\right)$, und kleine fast nadelförmige Kryställchen sind auf der Oberfläche der grösseren zerstreut resp. in anderen Mineralien als Einschlüsse enthalten.

Es wurden folgende Formen beobachtet:

$$
\begin{aligned}
& \text { am antilogen Pole } o\{11 T\}, \\
& \text { - analogen - } e\{0 T T\}, r\{T 00\}, c\{\text { TTT }\} .
\end{aligned}
$$

1) Einige rotthliche, im Britischen Museum befindliche Krystalle sind während eines vierjährigen Aufenthaltes in einem Glasschranke merklich verbleicht.

2) Einige Krystalle im Britischen Museum orreichen eine Länge von $12,5 \mathrm{~cm}$ und Kunz erwähnt sogar einen prachtvollen grünen Riesenkrystall von $25 \mathrm{~cm}$ Länge $\succ$ 2,5 cm Durchm. im Besitze des Herrn Bement in Philadelphia (18th Ann. Rep. U. S. Geol. Survey 1896-97, Part V (2), 1183, 1204; 20th Rep. 1898-99, Part VI (2), 600, Taf. I, Fig. E). 
Die Farbe der beiden Enden eines Krystalles steht scheinbar in keiner directen Beziehung mit der pyroëlektrischen Polarität desselben.

Es wurde von G. Rose ${ }^{1)}$ die Regel aufgestellt, dass dasjenige Ende, an welchem sich die Flächen des Hauptrhomboëders [trigonaler Pyramide] $\left(R R=46^{\circ} 54^{\prime}\right)$ auf den Kanten des trigonalen Prismas befinden, der antiloge Pol ist, und umgekehrt. Er fand aber, dass die Krystalle von Penig in Sachsen am antilogen Pole die Flächen von $R$ auf den Flächen des trigonalen Prismas zeigten, also von der obigen Regel eine Ausnahme bildeten.

Mit anderen Worten kann man diese Regel auf folgende Weise ausdrücken: Stellt man den Krystall mit dem antilogen Pole nach oben auf, so ist die häufigste Combination $+R\{100\}\{100\}$ mit dem Prisma $\{\overline{2} 11\}$ (resp. möglicherweise $-R\{22 \overline{1}\}\{\overline{\mathbf{2}} \overline{2} 1\}$ mit dem entsprechenden negativen Prisma \{2TT\}; obgleich, ohne directen Gegenbeweis, wohl das Vorhandensein der gleichen Formen stets anzunehmen ist). Eine etwaige Ausnahme von der Regel dürfte dann auf zweierlei Weise erklärt werden, entweder a) als eine Combination der selteneren negativen Pyramiden $-R\{22 T\}\{\overline{2} \overline{2} 1\}$ mit dem positiven Prisma $\{\overline{\mathbf{2}} 1 \mathbf{1}\}$, oder b) als Combination des selteneren negativen Prismas $\{2 T T\}$ mit $+R$.

Dass das negative Prisma am Turmalin nicht unbekannt ist, beweisen die Krystalle von Bovey Tracey, Sonnenberg im Harz und Gouverneur in New York, bei welchen die beiden trigonalen Prismen vorkommen, wovon das negative $\{\boldsymbol{z T} T\}$ in ersteren zwei Fällen zuweilen, im letzteren gewöhnlich vorherrscht ${ }^{2}$ ).

Die Ausnahme bei den Krystallen von Penig ist vielleicht (wie Rose zuerst vermuthete) auf Grund der ersteren Annahme zu erklären, weil bei diesen die $R$-Flächen sehr rauh und matt, die Flächen dagegen der die Kanten von $R$ abstumpfenden Pyramide $\frac{1}{2} R$ glatt und glänzend sind, während die Beschaffenheit der Flächen gewöhnlich das Umgekehrte zeigt. (Nach v. Worobieff ist jedoch diese Erklärung unwahrscheinlich.)

Die Untersuchung einer Anzahl der Krystalle von Haddam Neck durch die Kundt'sche Bestäubungsmethode zeigte, dass sich dieselben der obigen Regel anreihen. Bei ihnen wird auch ein weiterer Satz Rose's, dass die Basis nur an dem analogen Pole vorkommt, bestätigt.

Die Flächen von $r\{T 00\}$ sind stets glänzend; diejenigen von $e\{0 T T\}$

1) Pogg. Ann. 1836, 89, $285 ; 1843,59,357$.

2) Neulich hat V. v. Worobieff bei seiner umfassenden Untersuchung des Turmalin (diese Zeitschr. 1900, 38, 419) so viele Ausnahmen von der Rose'schen Regel gefunden, dass dieselbe nicht mehr als zutreffend angesehen werden kann. Bei den Krystallen von Haddam Neck sind zu wenige Formen vorhanden, um eine Bestimmung ihrer Polarität durch die von v. Wo roble ff vorgeschlagenen Hegeln zu ermöglichen; dennoch stimunt das Vorkommen von $o\{11 T\}$ am antilogen Po : mit seinen Beobachtungen iuberein. 
sind meist ebenso, zuweilen aber matt und drusig; $o\{11 T\}$ ist meist glänzend, aber etwas uneben.

Für den Flächenwinkel der Pyramide $o\{11 \bar{T}\}$ ergab sich als Mittelwerth aus vier Messungen $\left(\mathbf{7 6}^{\circ} 18^{\prime}-\mathbf{7 6}^{\circ} 45^{\prime}\right)$ an blassrothen Krystallen $\mathbf{7 6}^{\circ} 37^{\prime}$, woraus sich der Axenwinkel $a=114^{0} 4^{\prime}$ berechnet. Zwei Messungen an grünen Krystallen ergaben $76^{\circ} 4^{\prime}$ für den Flächenwinkel, $\alpha=114^{\circ} 0^{\prime}$ entsprechend.

Die hell gefärbten Krystalle sind meist nur schwach pleochroïtisch, während hei einigen der dunkelgrünen Exemplare der ordinäre Strahl fast vollständig absorbirt wird. Der Pleochröismus bei den verschiedenen Färbungen wird durch die folgende Tabelle veranschaulicht.

\begin{tabular}{|c|c|c|}
\hline \multirow[b]{2}{*}{ Farbe: } & \multicolumn{2}{|c|}{ Schwingungsrichtung } \\
\hline & $\begin{array}{c}\text { 川zur Axe } \\
\text { (extraordinärer Strahl): }\end{array}$ & $\begin{array}{c}\frac{1}{\text { zur Axe }} \\
\text { (ordinärer Strahl): }\end{array}$ \\
\hline rosaroth & tief rosaroth & rubinroth \\
\hline$-\quad-$ & rosaroth & blassroth \\
\hline - & sehr blasslila & bräunlich blassroth \\
\hline fast farblos & blassblau & - \\
\hline bläulichgrün & - & blass olivengrün \\
\hline $\begin{array}{ll}- & - \\
\text { graugrün } & \end{array}$ & $\begin{array}{l}\text { lauchgrün } \\
\text { graugrün }\end{array}$ & $\begin{array}{l}\text { [beinahe dunkel] } \\
\text { olivengrün }\end{array}$ \\
\hline hellgrün & blass grünlichblau & - \\
\hline iefgrün & grasgrün & [dunkel] \\
\hline violett & blass anilinroth & [beinahe dunkel] \\
\hline
\end{tabular}

Einige der >gleichmässigen, ziemlich blassgrünen * Krystalle sind von Penfield und Foote analysirt worden ${ }^{1}$, welche folgende Zahlen erhielten:

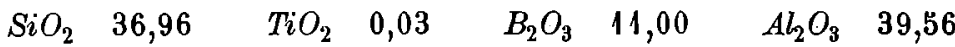

$$
\begin{aligned}
& \begin{array}{llllllll}
\mathrm{FeO} & 2,14 & \mathrm{MnO} & \mathbf{2 , 0 0} & \mathrm{MgO} & \mathbf{0 , 1 5} & \mathrm{CaO} & \mathbf{1 , 2 8}
\end{array} \\
& \begin{array}{llllllll}
\mathrm{Na}_{2} \mathrm{O} & 2,10 & \mathrm{Li} i_{2} \mathrm{O} & \mathbf{1 , 6 4} & \mathrm{H}_{2} \mathrm{O} & 3,10 & \mathrm{~F} & \mathbf{1 , 1 3}
\end{array} \\
& \text { Summe } \quad 101,09
\end{aligned}
$$

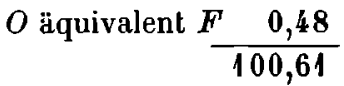

Flache tafelige Krystalle von schwarzem Turmalin (sowie dünne Prismen der grünen Varietät) kommen als Einschlüsse zwischen den Blättern des oben erwähnten farblosen Muscovits vor. In den »pockets s sind keine schwarzen Krystalle, obwohl einige der tiefgrünen Säulen auf den ersten Blick fast schwarz erscheinen.

.Nach $\mathrm{Kunz}^{2}$ ) sind eine Anzahl Krystalle gefunden worden, welche eine

1) Diese Zeitschr. 1899, 31, 33 з.

2) 19th Ann. Rep. U. S. Geol. Survey 1897-98, Part VI (z), 505. 
Von diesen sind $l, W, W^{\prime}$ neue Formen.

Die Form $l$ wurde fünfmal an vier Krystallen beobachtet als eine schmale Fläche auf der Kante $a / m$. Einer von diesen Krystallen zeigt ausserdem auch noch $\mu$, und zwar auf derselben Seiten von $a$ wie $l$. Wenn man nun $\mu$ als $\{3 \overline{2} T 1\}$ annehmen darf (diese Form soll häufiger sein wie $\left.\mu_{1}\{3 \bar{T} \overline{2} 1\}\right)$, so muss $l$ die Indices $\{7 \overline{4} \overline{3} 0\}$ besitzen. Für die complementäre Form $\{7 \overline{3} \bar{i} 0\}$ wurde kein Beweis gefunden.

Die Form $k^{\prime}$ wurde einmal beobachtet, ebenfalls als schmale Fläche auf der Kante $a / m$. An demselben Krystalle war auch $l$ vorhanden, aber auf der anderen Seite von $a$, also in der Zone [mhamlak' $m_{1}$. Jener Form muss demnach das Symbol $\{5 \overline{4} \overline{4} 0\}$ zukommen.

Von Haidinger 1 ) wurde eine Form $* f$ a an einem Krystalle vom St. Gotthard gefunden, welche nach seiner Figur und Beschreibung unserer Form $k^{\prime}$ entsprechen muss. Dieselbe wurde aber später von ihm aus der (sonst gleichen) Abbildung (Fig. 148, Taf. XXVII) in seiner Auflage von Mohs' Mineralogie (Edinburg 1825) ausgelassen, obgleich sie noch als $f(P+\infty)^{3} \mathrm{im}$ Formenverzeichnisse (Bd. II, S. 74) geblieben ist. Wohl aus diesem Grunde ist die Existenz derselben von Schrauf für zweifelhaft gehalten ${ }^{2}$ ) worden. Bei Des Cloizeaux ${ }^{3}$ ) ist die entsprechende Form ${ }^{2} h^{4}$ in die Abbildungen eingetragen, und zwar auf derselben Seite von $a$ wie $\mu$ an Krystallen von •Cornwall und auf beiden Seiten derselben Fläche an Krystallen von »Jumilla . Die Quelle dieser Figuren habe ich jedoch nicht entdecken können.

Die ergänzende Form $k$ ist von E. S. Dana an einem Krystalle von Paris, Maine ${ }^{4}$ ), gefunden worden. An den Krystallen von Haddam Neck scheint sie aber nicht vorzukommen.

Die Form $h\{3 \overline{2} T 0\}$ ist holoëdrisch entwickelt, wie an Krystallen von Poncione della Fibia im St. Gotthard von Kenngott $\left.{ }^{5}\right)$ beobachtet und von Klein $\left.{ }^{6}\right)$ bestätigt worden ist. Ebenso die neue Form $W\{7 \overline{5} \overline{2} 0\}$. Die holoëdrische Entwickelung ist an zwei grünlichen Krystallen nachgewiesen worden, welche folgende Flächen in der Prismenzone zeigen:

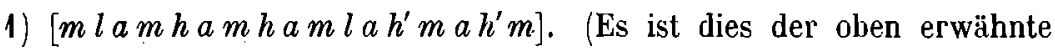
Krystall mit $\mu$.)

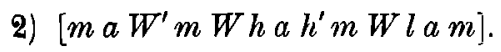

1) Edinb. Phil. Journ. 1824, 10, 148 und Taf. V, Fig. 16; Isis 1824, 852.

2) Ber. Akad. Wien 1870,62 (2), 754. D a n a (System 1892, 768, Ref. 4) erwahnt $k\{41 \overline{5} 0\}$ als von $\mathrm{Sch}$ rauf in Zweilel gezogen. Dieser aber wollte nur die Haiding e r'sche Form $\left(k^{\prime}\{14 \overline{5} 0\}\right)$ besprechen, obgleich seine Symbole alle beide umfassen.

3) Manuel de Minéralogie 2, 434 und Taf. LXXIII, Fig. 442, 443.

4) Amer. Journ. Sci. $1884(3), 27,480$ und diese Zeitschr. 9, 284.

5) Min. d. Schweiz 1866, 353.

6) N. Jahrb. f. Min., Geol. elc. 1871, 485. 
$W$ (resp. $W^{\prime}$ ) kommt auch an einem röthlichen Krystalle vor, in Combination nur mit $m$ und $a$. Es können hier die beiden Formen nicht unterschieden werden, wie es ebenfalls der Fall ist bei $h$ (resp. $h^{\prime}$ ) in derselben Combination an einem zweiten röthlichen Krystalle.

Der Nachweis der neuen, resp. zweifelhaften Formen beruht auf folgenden beobachteten Winkeln :

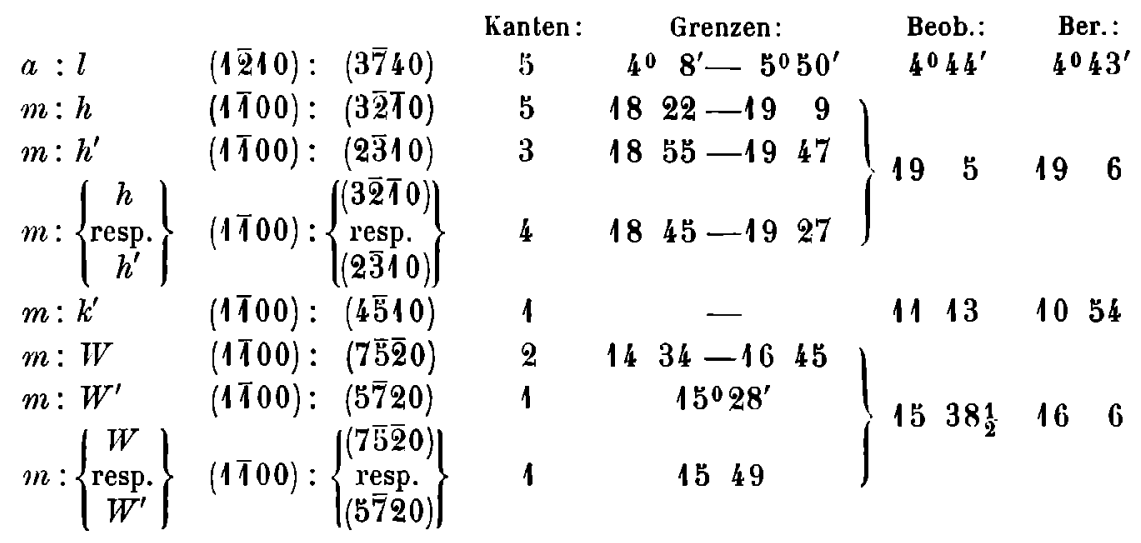

Die Basis ist sehr glänzend und liefert äusserst gute Reflexe; dem blossen Auge jedoch erscheint sie uneben, weil die Fläche von kleinen hexagonalen Vertiefungen (mit einem Durchmesser von $2 \mathrm{~mm}$ ) unterbrochen ist. Diese haben ihre Seiten parallel den Kanten $\mathrm{c} / \mathrm{m}$ und sind von etwas gekrümmten vicinalen Flïchen $(P)$ begrenzt, deren Indices nahe $\{2 . \overline{2} .0 .19\}$ liegen. Aehnliche Flächen, sowie solche einer Form $Q$ in der Nähe von $\{2 \overline{2} 07\}$ sind auch an den Kanten der Basis vorhanden.

Die Winkel derselben sind folgende:

\begin{tabular}{|c|c|c|c|c|c|c|}
\hline \multirow[b]{2}{*}{$:: P=(0001):(2 . \overline{2} .0 .19)$} & \multirow{2}{*}{$\begin{array}{c}\text { Kanten: } \\
5\end{array}$} & & & & \multirow{2}{*}{$\begin{array}{c}\text { Beobachtet: } \\
5^{0} \quad 4^{\prime}\end{array}$} & \multirow{2}{*}{$\begin{array}{c}\text { Berechnet } \\
5^{0} 61^{\prime}\end{array}$} \\
\hline & & & & & & \\
\hline$: Q=(0001):(2 \overline{2} 07)$ & 2 & 13 & $9-14$ & 8 & $1338 \frac{1}{2}$ & 1337 \\
\hline
\end{tabular}

- Die Flächen von $m$ sind glänzend und liefern gute Reflexe, diejenigen von $a$ sind schwach nach der Kante $a / m$ gestreift.

\section{Mikroklin.}

Der Mikroklin bildet dicke, grünlichweisse, undurchsichtige Krystalle (bis zu $5 \mathrm{~cm}$ Höhe und $4 \mathrm{~cm}$ Durchm.) vom gewühnlichen Habitus, welche oft sehr drusig und mit Vertiefungen besetzt sind, besonders auf den Flächen $e\{001\}$ und $x\{10 T\}$. Auf deren Oberfäche sitzen zuweilen kleine glänzende Kryställchen von Albit, sowie Krusten von Cookeït.

Es wurden folgende Formen beobachtet: $c\{001\}, b\{010\}, m\{110\}$, $M\{1 T 0\}, f\{130\}, \approx\{1 \overline{3} 0\}, x\{10 T\}, y\{20 \bar{T}\}, p\{\bar{T} 1 \mid\}, o\{\bar{T} 1\}$. 
Einige der Spaltungsstücke nach $c\{001\}$ zeigen die Mikroklinstructur, andere dagegen entbchren der Zwillingsbildung und besitzen einen $\Lambda$ uslüschungswinkel von ungefähr $14^{\circ}$. Der Auslüschungswinkel auf $b\{010\}$ ist etwa $+7^{0}$, und durch diese Fläche tritt eine positive Mittellinie schief heraus.

An einigen Krystallen ist auf $e\{001\}$ und $x\{101\}$ eine kreuzförmige Strcifung dem blossen Auge sichtlbar.

Die Stufe, welche weissen Beryll trägt, sowie eine Beryllstufe im Britischen Muscum zeigen eine pegmatitische Verwachsung von Quarz und weisse In Mikroklin.

\section{Albit.}

Die Krystalle dieses Minerals sind gross und gut ausgebildet; sie sind meisl tafelig nach $b\{010\}$, resp. nach $x\{$ T01\} ausgedehnt und dabei oft in paralleler resp. Zwillingsstellung zusammengewachsen auf die Weise, dass das Ganze einem grossen, nach der $b-\Lambda x e$ verlïngerten Krystalle ähnlich wird.

Gelegentlich kommen auch dicke, einfache Krystalle vor (bis $1 \mathrm{~cm}$ Durchmesser oder noch mehr) (Fig. 8, Taf. III).

Eine Verzwillingung nach dem Albitgesetze ist sehr häufig, sonst sind aber keine Zwillingsarten beobachtet worden.

Der Winkel der Spaltungsflächen $b: c$, aus einęm gemessenen Zwilling berechnet, ist $86^{\circ} 26 \frac{1}{2}^{\prime}$.

Fs sind folgende Formen vorhanden:

$$
\begin{aligned}
& b\{010\}, c\{001\}, \\
& m\{110\}, * Z\{120\}, f\{130\}, \subseteq\{150\}, M\{1 \bar{T} 0\}, \\
& x\{10 \bar{T}\}, r\{10 \overline{3}\}, y\{20 \bar{T}\}, \\
& u\{0 \overline{2} 1\}, \\
& u\{22 \overline{1}\}, \sigma\{44 \overline{3}\}, o\{11 \overline{1}\}, \delta\{1 \overline{1} 2\}, * X\{24 \overline{1}\}, \\
& g\{2 \overline{2} \bar{T}\}, p\{1 \bar{T}\}, * Y\{3 \bar{T}\}, * W\{111\} .
\end{aligned}
$$

\begin{tabular}{|c|c|c|c|c|c|c|}
\hline & & Kanten & Grenzen & Beob. & Ber. & \\
\hline$m: W$ & $(110):(111)$ & 1 & $\left\{\begin{array}{l}32029^{\prime} \text { bis } \\
3351 \text { (gewölbl) }\end{array}\right\}$ & $33010^{\prime}$ & $32028^{\prime}\{$ & $\left\{\begin{array}{c}\text { gewölbte Flăche an } \\
\text { der Kante } c / m \text {,(an } \\
\text { einem Krystalle) }\end{array}\right.$ \\
\hline$y: x$ & $(\overline{2} 01):\left(\overline{2} \bar{T}_{1}\right)$ & 2 & $520: 57^{\prime}-330 \leqslant 0^{\prime}$ & $\begin{array}{ll}53 & 18.1 \\
2\end{array}$ & 5256 & \\
\hline$u: X$ & $(\overline{2} \overline{2} 1):(\overline{3} 11)$ & 1 & - & $19: 31^{\circ}$ & $20 \quad 7 \frac{1}{2}$ & schmale Fláche an \\
\hline$e: X^{\prime}$ & $(001):(\overline{2}-11)$ & 1 & - & $87 \quad 38$ & $8752^{2}$ & der Kanle $b / u$ (an \\
\hline$b: \boldsymbol{X}$ & $(010 !:(24 \overline{1})$ & 1 &.- & 398 & 3924 & schmale glänzende \\
\hline$y: Y^{\prime}$ & $(\overline{2} 01):(\overline{31} 1)$ & 1 & - & 1612 & 1618 & $\begin{array}{l}\text { Fläche an der Kante } \\
y / M \text { (an einem } \\
\text { krystalle) }\end{array}$ \\
\hline$b: Z$ & $(010):(120)$ & 1 & - & {$\left[\begin{array}{ll}40 & 46\end{array}\right.$} & 4121 & $\begin{array}{l}\text { sehr. schmale Flache } \\
\text { in der Zone [bm] } \\
\text { (an einem Kry- } \\
\text { stalle) [Einstellung } \\
\text { durch maximale } \\
\text { Helligkeit] }\end{array}$ \\
\hline
\end{tabular}


Von diesen sind $W, X, Y, Z$ scheinbar noch nicht beobachtet worden. Ihre Existenz ist aus den vorstehenden Messungen auf S. $1 / 4$ zu folgern. Die Mittelwerthe der Auslüschungswinkel sind

$$
\text { auf } \begin{aligned}
c(001) & =3 \frac{1}{2}, \\
b(010) & =19 \frac{1}{2} 0^{0}
\end{aligned}
$$

Schüne Stufen, bestehend aus Aggregaten von dünnlafeligen Krystallen mit Cookeït überzogen, werden ebenfalls gefunden.

\section{Beryll.}

Die Sammlung in Oxford besitzt vier Exemplare von Beryll, welche zweierlei Typus zeigen. Den ersten Typus stellen drei vollkommene und meist nur durchscheinende Krystalle dar, welche aus einer blass rosarothen (zuweilen durchsichtigen) äusseren Hülle mit grünlichweissem Kerne bestehen. Es sind hexagonale Säulen $m\{10 \bar{T} 0\}$, mit der Basis am Ende und Flächen einer Pyramide $s\{1 \mid \overline{2} 1\}$ auf den Ecken. Die Flächen sind alle rauh und matt, mit der einzigen $\Lambda$ usnahme eines kleinen Stückes rosa durchsichtigen Materials an einem der vier Krystalle, welches eine sehr glänzende Ebene $m$ trägt. Die Exemplare erreichen $8 \mathrm{~cm}$ im Durchmesser und zeigen in auffallender Weise eine ziemlich vollkommene Spaltbarkeit nach $m\{10 \overline{0} 0\}$ neben der gewöhnlichen (hier ebenfalls guten) nach $c\{0001\}$. Sie enthalten zuweilen Krystalle von Albit und Turmalin.

Dem zweiten 'Typus gehürt ein fast farbloser Krystall von elwa $2 \mathrm{~cm}$ Durchmesser, in grauen Quarzkrystallen eingelagert, an. $\mathbf{E r}$ entbehrt fast vollkommen glatter Flächen und jst daher schwer zu entziffern. Durch den Vergleich jedoch mit dem unten beschriebenen Krystalle im Britischen Museum erkennt man dessen beide glänzenden Flächen als $m\{10 \overline{1} 0\}$, während die übrigen Flächen wohl $s\{11 \overline{2} 1\}, n\{31 \overline{4} 1\}, p\{10 \overline{1}\}$ angehüren. Die Basis ist wahrscheinlich auch vorhanden, sie ist aber von den umgebenden Quarzkrystallen fast vollständig bedeckt. Der Habitus ist von dem des in Fig. 9, Taf. III abgebildeten Krystalles etwas abweichend, weil wegen Verkürzung des Prismas die Flächen von $m\{10 T 0\}$ auf kleine von $n\{31 \overline{4} 1\}$ begrenzte Rauten reducirt sind.

Im Britischen Museum befinden sich zwei Beryllexemplare von Haddam Neck, welche beide als "Cäsiumberyll e etiquettirt sind ${ }^{1}$. Von diesen ist das eine ein grosses dem ersten Typus angehörendes dickes Prisma von einer sehr blass rosa Farbe, welches kleine Krystalle des gewöhnlichen Turmalins und ein Stückchen eines braunen Minerals (wohl Granat) einschliesst.

Das andere, welche den zweiten Typus darstellt, ist ein fast farbloses hexagonales Prisma (Fig. 9) von $1,3 \mathrm{~cm}$ Durchm. und $2,5 \mathrm{~cm}$ Länge mit

1) Herr Lazard Cahn, durch den die stuicke erhalten wurden, theilt mit. dass diesclben auf Cäsium von Prof. Penfield geprüft worden sind. 
Pyramidenflächen und wird von grauem Quarz, weissem Mikroklin, rothem und grünem Turmalin und Albit begleitet, auf einem dem "Schriftgranit* ähnlichen Mikroklinpegmatit. Die Flächen gehören folgenden Formen an:

$\begin{array}{cc}\text { Dana: } & \text { Miller: } \\ m\{10 \overline{1} 0\} & a\{10 \bar{T}\} \\ c\{0001\} & o\{111\} \\ s\{11 \overline{2} 1\} & r\{100, \bar{T} 22\} \\ n\{31 \overline{4} 1\} & \{20 \overline{3}, 42 \overline{3}\} \\ p\{10 \bar{T} 1\} & p\{120\} .\end{array}$

Die Basis ist sehr uneben, mit Rinnen und Vertiefungen, aber glänzend; das Prisma ist glatt und glänzend, und von den Pyramiden ist $s$ etwas mit Vertiefungen besetzt, während $n$ meist matt, aber stellenweise glänzend ist. Die letztgenannten Flächen sind manchmal gegen $s$ zu abgestumpft: $p$ ist nur als kleine dreieckige Fläche auf den Ecken $s c s$ vorhanden.

Dieser Krystall ist dem farblosen Beryll von Goshen in Massachusetts (dem sogenannten •Goshenit «) sehr ähnlich.

\section{Quarz.}

Der Quarz kommt in fast farblosen, sowie in etwas rauchigen Krystallen vor, wovon erstere auf eigenthümliche Weise nach einem prismatischen Flächenpaare tafelig verzerrt sind. Solche Krystalle sind nur auf einer Seite regelmässig entwickelt, während auf der anderen Seite die Flächen unterbrochen, uneben und drusig sind, so dass das ganze Stück (obgleich oft an beiden Enden entwickelt und ringsherum von Flächen begrenzt) das Aussehen eines von der Seite eines regelmässigen Krystalles abgebrochenen Splitters darbietet.

Die Rauchquarzkrystalle zeigen den gewöhnlichen Habitus, wobei den beiden die Pyramide bildenden Rhomboëdern zuweilen eine verschiedene Grösse zukommt.

Die Oberfläche der Krystalle ist oft mit Krusten von Cookeït bedeckt.

\section{Cookeït.}

Es werden kleine kugelförmige Massen (bis zu 5 mm Durchmesser, die meisten aber nicht $1 \mathrm{~mm}$ überschreitend) gefunden, welche folgende Eigenschaften besitzen.

Die Kügelchen zeigen eine gelblichweisse Farbe und sind aus radialen silberglänzenden Blättern von sechsseitigem Umrisse zusammengesetzt. Unter dem Mikroskope zeigen die letzteren eine radiale Eintheilung in dreieckige, zweiaxige Felder, welche je senkrecht zur Seite des Sechseckes gestreift sind und die senkrecht austretende positive Bisectrix eines grossen Axenwinkels, dessen Ebene senkrecht zur Streifung, erkennen lassen. Gegen die Mitte 


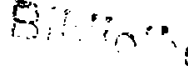

Ueber das Mineralvorkommen zu Haddam Neck in Connẹcticut (Ver. St.). 117

des Sechseckes hin nimmt der Axenwinkel ab und der innerste Theil ist i einaxig mit senkrecht stehender Axe und schwacher positiver Doppelbrechung. Diese Eigenschaften stimmen mit den von Penfield 1) für den Cookeït angegebenen genau überein.

Der Cookeït befindet sich hauptsächlich an der Oberfläche der Albitund Quarzkrystalle und der Lepidolithsäulen. Auf dem Turmalin ist er nicht häufig, und auch dann ist die unterliegende Oberfäche des Turmalin vollkommen frisch und glatt; daher ist der Cookeït hier wohl nicht ein ¿Zersetzungsproduct des Rubellit", wie Brush $^{2}$ ) in Bezug auf den Cookeït von Hebron und Paris in Maine vermuthet hat.

Wegen der innigen Mischung der verschiedenen Mineralien lässt es sich kaum entscheiden, aus welchem von ihnen der Cookeï entstanden sei; es sind aber vielleicht die Glimmer als wahrscheinlichste Muttersubstanzen anzusehen.

Vor dem Löthrohre stimmen die Eigenschaften mit denen des Cookeït überein.

\section{Flussspath.}

Der Flussspath ist tief röthlichbraun und zeigt zuweilen Flächen des Oktaëders. Diese Stücke sind sämmtlich lose. Ausserdem habe ich neulich ein Exemplar gesehen, welches aus kleinen, blassgrünen, undurchsichtigen und etwas drusigen Oktaëdern ( $4 \mathrm{~mm}$ Durchm.) besteht und zum Theil eine violette Farbe zeigt, in Association mit Kügelchen des Cookeït und Fasern von rosarothen Muscovit.

\section{Mikrolith.}

Von Mikrolith habe ich keine Exemplare untersuchen können, weil weder in der Sammlung zu Oxford, noch im Britischen Museum dieses Mineral vorhanden ist. Nach einer Mittheilung des Herrn Schernikow wurde er > found sparingly, associated with the other minerals. A few good crystals, perhaps $\frac{1}{4}$ inch $(0,625 \mathrm{~cm})$ in diameter, were obtained, but most of them were broken «.

Herr L. Cahn hat mir geschrieben, dass das Mineral von Herrn Prof. Penfield als Mikrolith bestimmt wurde, und dass die Krystalle in dem rosarothen Beryll resp. in Albit vorkommen.

\section{Colnmbit.}

Der Columbit soll ebenfalls in den meisten Steinbrüchen zu Haddam Neck, in Association mit Beryll, gefunden worden sein. Ich habe davon zwar keine Exemplare gesehen, aber die Krystalle werden von Hrn. Schernikow beschrieben als klein, jedoch gut ausgebildet, mit sehr glänzenden Flächen,

1) Amer. Journ. Sci. 1893 (3), 45, 393. Ref. diese Zeitschr. 25, 99.

2) Amer. Journ. Sci. 1866 (2), 41, 246. 
Sie unterscheiden sich wohl dadurch von dem bekannten Columbit von Haddam, wo die Krystalle meist gross und rauh sind.

\section{Vorkommen und paragenetische Verhältnisse.}

In Bezug auf ihr Vorkommen und ihre Eigenschaften zeigen einige der Mineralien von Haddam Neck eine auffallende Aehnlichkeit mit denen der bekannten Vorkommnisse in den Pegmatitgängen von. Maine und anderen Orten Neu-Englands. Die grossen, mit einem Lepidolithsaume umgebenen Muscovitkrystalle z. B. entsprechen genau solchen von Auburn, und dazu zeigen einige der letzteren, ausserhalb des Lepidolith, ein wenig rhombischmosaikartigen Glimmer, welcher dem, für die Stufen von Haddam Neck einen so bedeutenden Grundzug bildenden, rosarothen faserigen Muscovit vollkommen ähnlich ist.

Auch die regelmässige Verwachsung von grünlichweissem Muscovit mit derbem Quarz, welche an mehreren der Stufen von IIaddam Neck zu beobachten ist, hat ihr genaues Analogon in einem von Goshen in Massachusetts stammenden Exemplare im Britischen Museum, welche auch Beryll trägt. Der auf derselben Stufe vorkommende Beryll (der farblose »Goshenit «) gleicht auch in auffallender Weise dem betreffenden Mineral von Haddam Neck, dessen Cãsiumgehalt eine Verwandtschaft auf der anderen Seite mit den Beryllen von Norway und Hebron in Maine anzejgt, welche nach Penfield und Harper resp. nach Wells einen Gehalt von 1,66 bis $3,6 \%$ $\mathrm{Cs}_{2} \mathrm{O}$ besitzen.

Soweit man aus der relativen Lage von Haddam und Haddam Neck schliessen kann, und nach den mir zugänglichen Nachrichten darüber scheint es wahrscheinlich, dass die den Gneiss durchsetzenden Pegmatitgänge, in welchen sich die Mineralien der beiden Vorkommnisse befinden, demselben Gangsysteme angehören, obgleich sich die Mineralien aus den verhältnissmässig neu eröffneten Feldspathbrüchen zu Haddam Neck einigermassen von denjenigen aus den älteren Fundorten, die man unter dem Namen Haddam zu vereinigen pflegt, unterscheiden. Die Turmaline z. B, von Haddam sind dicke, schwarze Säulen; auch ist der von Haddam bekannte Chrysoberyll zu Haddam Neck noch nicht gefunden worden, obwohl er auf beiden Ufern des Flusses vorgekommen sein soll ${ }^{1}$ ).

Was die Bildungsfolge der Mineralien betrifft, so kann man nicht viel sagen, weil die verschiedenen Arten auf so innige Weise durcheinander gewachsen sind, dass sie erscheinen, als ob sie ziemlich gleichzeitig entstanden sein müssten. Unter den letztgebildeten Mineralien war wohl der Quarz, weil er oft ganze Krystalle von Turmalin und Massen des faserigen Muscovits vollkommen einschliesst. Zuweilen bildet er eine pegmatilische Ver-

1) Amer. Journ. Sci. 1822 (1), 4, 33; 1823 (1), 6, 220. 
wachsung mil Mikrokilin und enthält zuweilen auch Blïttchen des grünlichweissen Muscovits, welche unter sich parallel liegen, so dass dadurch eine etwas ähnliche Structur entsteht.

Die Glimmer stellen vielleicht eine frühe Stufe der Krystallbildung dar. Unter sich weichen sie niemals von der lieihenfolge: grünlichweisser Muscovit - Lepidolill - faseriger Muscovit, ab. Kleine Lepidolithschüppchen, welche in den Apatilkrystallen enthalten sind, werden nach Auflösung der letzteren in Süure zurückgelassen. Der Mikroklin ist wohl eine spätere Bildung, etwa gleichzeitig mit dem Quarz. Er enthält zuweilen Turmalin und Lepidolith.

Zum Schlusse möchte ich meinen herzliclısten Dank aussprechen dem Herrn Schernikow, der mir Nachricht über den Fundort und die Art des Vorkommens freundlichst geliefert hat, Herrn Prof. Berwerth dafür, dass er mir die Glimmersammlung im Wiener llofınuseum durchzusehen erlaubt hat, sowie Herrn L. Fletcher für die gleiche Erlaubniss die Mineralien im Britischen Museum belreffend.

Auch will ich hier Herın Prof. Penfield danken für freundliche Winke bei der Inalyse und besonders Herrn l'rof. Miers, der mich während der ganzen Arbeit auf's Freundlichste berathen hat.

\section{Erklärung der Figuren.}

Fig̣. 1. Spaltungsblättchen des rosarothen, mosaikartigen Muscovils, die Anordıung der Rhomben in Zwillingssslellung zoigend (Vergröss. $\times 14$ ).

Fig. 2. Eine laser des rosarothen Muscovits. $\pi\{552\}, S\{\overline{551}\}$. (Die Abweichung der Flächen von der Gestalt eines Prismas ist elwas iibertrieben.)

Fig. 3 und 4. Blättchen von Krystallen von Muscovit mil Lepidolithsaum ( $M$ Muscovit, $L_{m}$ makrodiagonale' und $L_{b}$ brachydiagonaler Lepidolith;. Dic verschiedenen Parlien sind je nach ihren Axenebenen schrafirt. Nichl schraffirte Parlien des Saumes löschen wegen der Verzwilligung nicht aus.

Fig. 3 zeigt cin schmales Band von $L_{m}$ in paralleler stellung um $M$ mit draussen befindlichen $L_{l}$-Partien (Vergr. $\times 7$;

Fig. 4 zeigt Partien von $L_{m}$ in paralleler Stellung und von $L_{b}$ in Zwillingsstellung um $M$ 'Vergr. $\times 13\} . \quad m\{110\}, b\{010\}$.

Fig. :̈. Blättchen von einem Krystalle makrodiagonalen Lepidoliths, die Anordnung des faserigen Muscovitsaumes zeigend. $m\{110\}, b\{010\}, Q\{130\}$. Die mit II bezeichneten Partien sind in der Auslöschungsstellung (Vergr. $\left.\times 5 \frac{1}{2}\right)$

Fig. 6. Quergebänderte Turmalinsäule (Länge $30 \mathrm{~mm}$, Durchm. : $\mathrm{mm}$ ). $0\{11 \overline{1}$.

Fig. 7. Stereographische Projection des Apatits, sämmtliche an den Krystallen von Haddam Neck beobachtete Formen enthaltend.

Fig. 8. Albit $\{b\{010\}, c\{001\}, m\{110\}, Z\{120\}, f\{130\}, \zeta\{150\}, M\{1 \overline{1} 0\}, x\{10 \bar{T}\}, r\{40 \overline{3}\}$,

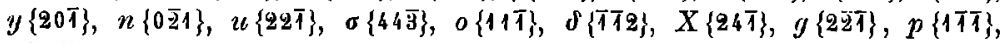
$Y\{3 \overline{1} \bar{T}\}, W\{111\})$.

Fig. 9. Beryll $\{m\{10 \overline{1} 0\}, c\{0001\}, s\{11 \overline{2} 1\}, n\{31 \overline{1} 1\}, p\{10 \overline{1} 1\}$. 